\title{
Do Haemostasis Activation Markers that Predict Cardiovascular Disease Exist?
}

\author{
Mojca Stegnar, Nina Vene, Mojca Bozic
}

Department of Angiology, University Medical Centre, Ljubljana, Slovenia

\author{
Key words \\ Cardiovascular risk - Hypercoagulability . \\ Haemostasis activation markers - D-dimer . \\ Prothrombin fragment 1+2 . Fibrinopeptide A . \\ Soluble fibrin - Thrombin-antithrombin complex . \\ Plasmin-antiplasmin complex
}

\begin{abstract}
Recognition of the central role of thrombosis in the pathogenesis of cardiovascular disease has prompted growing interest in the association of haemostatic variables with cardiovascular disease. In investigating the predictive value of haemostasis markers, a promising type of measurement is that of the activation products of coagulation and fibrinolysis: prothrombin fragment 1+2 (F1+2), fibrinopeptide A (FPA), soluble fibrin, thrombin-antithrombin (TAT), plasmin-antiplasmin (PAP) complexes and D-dimer. D-dimer was most extensively studied and there is substantial evidence that D-dimer is a strong, consistent predictor of cardiovascular events in the general population and in patients with cardiovascular disease. Data on other markers are considerably less abundant and more controversial. The prognostic value of these markers remains to be fully defined in future epidemiological and clinical studies.
\end{abstract}

Copyright @ 2004 S. Karger AG, Basel

\section{KARGER}

Fax +4161306 1234

E-Mail: karger@karger.ch

www.karger.com

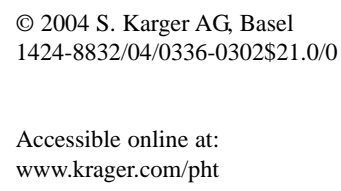

\section{Introduction}

The pathophysiology of atherothrombosis in cardiovascular disease is complex and multi-factorial. Rupture of an atherosclerotic plaque with subsequent thrombus formation on the site of plaque rupture is now universally accepted as the final pathophysiological mechanism leading to acute ischemic cardiovascular events. Thrombosis plays an important role not only in the pathophysiology of acute ischemic cardiovascular events but also in the process of atherogenesis, especially in the progression of the disease. Haemostatic factors are involved in the initiation of early atherosclerotic lesions, contribute to the progression of plaque growth and may lead to deleterious clinical events if a hypercoagulable state is present at the time of plaque fissuring or rupture [1].

Recognition of the central role of thrombosis in the pathogenesis of cardiovascular disease has prompted growing interest in the association of haemostatic variables with this disease. The observation that the mean level of some haemostatic factors was higher, or the level of coagulation inhibitors was lower in subjects who developed cardiovascular disease led to the concept of a hypercoagulable state in this disease. The beneficial effects of antiplatelet, anticoagulation or thrombolytic therapies in prevention and treatment of ischemic cardiovascular disease support the role of thrombosis and hypercoagulability in the initiation and progression of the disease.

Prof. Mojca Stegnar

Department of Angiology

University Medical Centre

1525 Ljubljana, Slovenia

Tel: +386 1522 8052; Fax: +386 1522 8070; E-mail: Mojca.Stegnar@trnovo.kclj.si 
Based on these considerations, a variety of epidemiological and clinical studies have been carried out to investigate the ability of haemostatic variables and new markers of haemostasis activation to improve risk prediction of future cardiovascular events. There are two main types of measurements in the investigation of the hypercoagulable state in cardiovascular disease: (i) measurement of levels of haemostatic factors and (ii) measurement of activation products. In the former type of measurements the most promising factors identified have been fibrinogen, factor VIII, von Willebrand factor and the fibrinolytic variables - tissue-type plasminogen activator (t-PA) and t-PA inhibitor-1 (PAI-1). During activation of haemostasis a variety of substances can be identified: peptides released during activation of coagulation factors, complexes of activated haemostatic factors with their inhibitors and degradation products of fibrin. Substances released or formed during activation of the haemostatic system are termed activation markers, because they indicate that haemostasis activation has taken place [2]. This review focuses on activation markers of coagulation and fibrinolysis: prothrombin fragment 1+2 (F1+2), thrombin-antithrombin (TAT) complexes, fibrinopeptide A (FPA), fibrin monomer/soluble fibrin, D-dimer and plasminantiplasmin (PAP) complex.

In the majority of clinical studies on the diagnostic and predictive value of haemostasis activation markers, only one of these markers has been tested. Although several of them have been shown to predict risk independently of conventional risk factors, the question remains which marker should be preferred in a routine clinical setting, or which cluster of variables reflecting different pathophysiological aspects of atherothrombosis should be used. Besides analytical and technical considerations, the main reason in favour or against a given marker consists in its independence of other markers reflecting the same pathophysiological pathway.

\section{Blood Coagulation and Fibrinolysis Activation Markers}

During conversion of prothrombin to thrombin by factor $\mathrm{Xa}$, a degradation product of prothrombin (F1+2) arises. Plasma F1+2 is a peptide of 32 amino acids and has a plasma half life of 10-90 min. It reflects the enzymatic activity of factor Xa on prothrombin, and is thus a marker of thrombin generation [3].

Thrombin released by activation of prothrombin is inactivated by antithrombin, forming a thrombin-antithrombin (TAT) complex. The TAT complex has a molecular weight of $88 \mathrm{kD}$ and elutes as a single peak from a sodium dodecyl sulphate gel [4]. The level of the TAT complex expresses

Haemostasis Activation Markers as Predictors of Cardiovascular Disease the amount of thrombin present in circulation and is therefore considered to indicate hypercoagulability.

In the process of converting fibrinogen to fibrin monomers by thrombin, fibrinopeptide A (FPA) and fibrinopeptide $\mathrm{B}$ are derived from the $\mathrm{N}$-terminal part of the $\mathrm{A} \alpha$ and $\mathrm{B} \beta$ chains of fibrinogen and can be measured as indicators of thrombin activity. Human FPA is a 16 amino acid peptide with a molecular weight of 1540 and a very short plasma half-life (3-5 min). Since the fibrinogen molecule has a dimeric structure, two identical FPA molecules can be cleaved from each fibrinogen molecule [5].

After release of FPA from fibrinogen, the resulting fibrin monomers display an intense tendency to polymerise. Fibrin monomers may polymerise not only with other fibrin monomers, but also with fibrinogen and various fibrinogen and fibrin degradation products, forming soluble fibrin [6]. Therefore, in plasma fibrin monomer is present only as a component of soluble fibrin complexes.

Polymerised fibrin monomers form soluble non-crosslinked fibrin, which is then cross-linked by factor XIIIa to form a stable, lysis-resistant insoluble fibrin clot. Degradation of cross linked fibrin is achieved by activation of the fibrinolytic system on the surface of fibrin to which tPA and plasminogen are bound. Fibrin acts as a cofactor in plasminogen activation induced by t-PA. Combination of bound t-PA and plasminogen generates plasmin, which degrades fibrin to fibrin degradation products. These contain the D-dimer fragment, which retains cross links of the original fibrin [7]. Level of D-dimer can be regarded as a marker of cross-linked fibrin turnover and suggest that the formation of thrombin due to activation of the coagulation system and plasmin as a result of activation of the fibrinolytic system has taken place. Moreover, D-dimer is not only a marker of ongoing degradation of cross-linked fibrin, which may occur in response to thrombus formation, but may also be directly involved in atherogenesis [8]. D-dimer present in the atherosclerotic lesion stimulates smooth muscle cell proliferation and is chemotactic for monocytes. It may also increase the synthesis of fibrinogen by stimulation release of interleukin-6 from monocytes, which in turn stimulates fibrinogen synthesis in the liver.

When plasmin is generated in the circulation, the potent plasmin inhibitor, antiplasmin, immediately inhibits its activity and measurement of plasmin-antiplasmin (PAP) complex has been shown to be an adequate approach to assessing the amount of plasmin formed in vivo [9].

Pathophysiol Haemost Thromb 2003/2004;33:302-308 


\section{Population Studies on Activation Markers and Cardiovascular Risk}

All individuals have a measurable amount of activation markers in their circulation, confirming a Astrup's original hypothesis that low grade coagulation and fibrinolysis are a continuous processes of wear and tear in normal individuals [10]. During systemic or even local activation of coagulation and fibrinolysis, increased amounts of activation products are generated. Therefore, measurement of activation markers has been proposed as a method of assessing the hypercoagulable state of an individual.

In a cross-sectional study the presence of a hypercoagulable state as assessed by an increased level of activation markers was investigated by Van der Bom and co-workers [11] in patients with stable atherothrombotic cardiovascular disease. From a population-based cohort they randomly selected subjects with a history of myocardial infarction, stroke and/or transient ischemic attack and peripheral arterial disease. In all three manifestations of arterial disease there were signs of increased formation and degradation of fibrin as reflected by increased D-dimer. The increased level of Ddimer has also consistently been reported in other studies on subjects with stable arterial disease [8,12,13]. Findings from studies on a possible association between activation of prothrombin and plasminogen as judged from increased levels of F1+2, TAT and PAP and arterial disease are less evident $[11,14]$.

D-dimer was shown to be a strong, consistent predictor of cardiovascular events (cardiovascular death, myocardial infarction, stroke, leg ischemia, arterial surgery and post surgery arterial occlusion) in several prospective studies. Danesh and co-workers [15] performed a meta-analysis of previous relevant prospective studies in subjects with and without pre-existing cardiovascular disease. In a comparison of men in the top third compared with those in the bottom third of D-dimer levels, a combined analysis yielded an odds ratio for coronary heart disease of 1.7. D-dimer level was not associated with smoking, blood lipids, blood pressure, and other risk factors. The potential value of D-dimer in coronary heart disease prediction was also evaluated in the Caerphilly Study. D-dimer was s strong, independent predictor of coronary events and it was suggested that it may also add to the predictive value of major conventional risk predictors for cardiovascular disease [16].

Data on the predictive value of other coagulation and fibrinolysis markers is less consistent. In the Caerphilly study F1+2 and TAT were evaluated, but neither of them was associated with incident ischemic heart disease [16] nor could Folsom and co-workers [17] establish an association between incident coronary heart disease and F1+2 in stored baseline plasma samples of subjects who developed coro- nary heart disease. Cushman and co-workers [18] studied PAP for the prediction of arterial thrombosis in healthy elderly persons without cardiovascular disease. PAP levels predicted future myocardial infarction and the relationship was independent of other risk factors, including inflammation markers.

\section{Activation Markers in Acute Coronary Syndromes and Assessment of Early and Long-term Outcomes}

Acute coronary syndromes represent a spectrum of disease from unstable angina pectoris to acute myocardial infarction which share a common pathophysiological mechanism: (i) rupture of an atherosclerotic plaque, (ii) activation of haemostasis and (iii) formation of a completely or incompletely occluding thrombus at the site of the coronary plaque rupture. During the acute event procoagulant, fibrinolytic and inflammatory pathways are activated in most patients. Yet it seems that the activation of haemostatic system is not confined to the acute phase but may also play an important role in chronic progression of atherosclerotic coronary disease. Ongoing thrombogenesis thus appears to be an important determinant of short- as well as long-term prognosis, and measurements of coagulation activation markers may possibly identify high-risk patients who will develop complications.

In patients with unstable angina and acute myocardial infarction increased levels of F1+2 and TAT during hospitalization were reported [19,20]. Even more important is the consideration that elevated levels of markers of thrombin generation in a high proportion of these patients persist several months after the symptomatic period [21]. This hypercoagulability far beyond the acute phase inevitably increases the risk of thrombotic complications, manifested as recurrent angina and an increased risk of subsequent major cardiac events in the first months after the acute episode [21,22].

Another reliable marker of thrombin activity, FPA, has also been found to be abnormally high in the majority of patients with unstable angina in its active phase [21,23], being the greatest in patients showing ST-segment changes [24] and in those with angiographically detectable intracoronary thrombosis [25]. However, in these studies a significant minority of patients did not have increased FPA despite the presence of an angiographically visible thrombus, or had increased FPA in the absence of a visible intracoronary thrombus. Increased levels of FPA have also been documented in patients with unstable angina who developed acute myocardial infarction during hospitalization [26] and were associated with increased risk of subsequent cardiac death [27]. 
Increased level of D-dimer has also been observed in patients with acute coronary syndrome. The advantage of Ddimer is in its earlier rise than conventional cardiological injury markers (creatine kinase, creatine kinase-MB isoenzymes, cardiac troponins and myoglobin). Thus it may represent a novel early marker of the thrombotic process leading to acute coronary syndrome, and may predict myocardial ischemia before myocardial necrosis occurs. A study by Bayes-Genis and co-workers [28] evaluating patients with acute chest pain suggested that plasma levels of D-dimer might have an additional role in a diagnostic model of myocardial infarction detection and increase the diagnostic sensitivity of the electrocardiogram and history. Unfortunately, levels of troponins were not included in the model. In addition, marked increases in circulating D-dimer are indicative of recurrent angina [29] and other thrombotic complications in patients with acute coronary syndromes [30].

Besides its role in acute coronary syndrome, chronically elevated level of D-dimer emerged as a powerful independent predictor of long term recurrent coronary events. Ddimer, measured by ELISA, has been shown to correlate well with subsequent acute coronary events, either non-fatal myocardial infarction or cardiac deaths, after an index acute myocardial infarction [31,32]. Studies documented that semi quantitative D-dimer assays such as the latex agglutination test or the whole blood agglutination test also produce comparable results to standard ELISA, showing a significant, concentration-dependent prediction of recurrent coronary events [31,33].

Among other coagulation markers the long-term prognostic potential of soluble fibrin among patients with coronary artery disease was investigated by Hetland and coworkers [34] who measured soluble fibrin in plasma samples of patients with acute chest pain admitted consecutively to hospital. Outcome analysis during the following 30 months after the index event revealed higher levels in those with coronary re-events or death than in patients without new events. The prognostic value for death or re-infarction of soluble fibrin was confirmed in patients with myocardial infarction by Dempfle and co-workers [32].

\section{Predictive Value of Activation Markers in Atrial Fibrillation}

Chronic nonvalvular atrial fibrillation is associated with an increased risk of thrombotic cardiovascular events even during oral anticoagulant therapy. The increased risk of stroke is usually due to embolization of a thrombus formed within the left atrial appendage. Other cardiovascular events often encountered in these patients, such as acute myocar-

Haemostasis Activation Markers as Predictors of Cardiovascular Disease dial infarction, progression of heart failure, progression of vascular disease and cardiovascular death, are in general also the consequence of intracardiac or intravascular thrombus formation [1].

Elevated levels of the plasma markers of coagulation activation - F1+2, TAT and fibrin D-dimer - have been reported in patients with atrial fibrillation [35,36] and are significantly decreased during oral anticoagulant treatment [36,37], probably reflecting suppression of in vivo thrombin formation and fibrin turnover. According to these data, F1+2, TAT and D-dimer might have additional roles as markers of the clinically relevant antithrombotic effects of oral anticoagulant treatment.

The predictive value of F1+2, TAT and D-dimer in patients with atrial fibrillation during oral anticoagulant treatment has been studied recently [37]. In patients who suffered cardiovascular events a significantly higher level of D-dimer, both at baseline and during oral anticoagulant treatment was observed, possibly reflecting the persistence of a high fibrin turnover even during adequate oral anticoagulant treatment. The hazard of cardiovascular events increased with increasing plasma D-dimer concentration and was about five-fold greater in the highest D-dimer quartile than in the lowest two quartiles. The predictive value of D-dimer was independent of the main clinical and echocardiographic risk factors for adverse outcomes. On the other hand, levels of TAT and F1+2 were not associated with thromboembolic events.

\section{Activation Markers and Stroke}

Stoke is a thrombotic process in at least $80 \%$ of cases, the final precipitating event being the formation of an occlusive thrombus in the intra-cerebral arteries. Brisk activation of coagulation, increased thrombin generation and fibrin turnover, as well as altered fibrinolytic activity, have been demonstrated in patients with acute stroke [38]. Haemostatic abnormalities are associated with stroke severity and early progression of the disease [39]. Recent studies have also shown that haemostasis activation differs among stroke types. The highest levels of haemostasis activation markers, particularly D-dimer, are found in patients with cardio-embolic stroke [38,40], who have higher mortality rate than those with noncardioembolic stroke [41]. Patients with lacunar infarctions with low mortality rate have normal levels of haemostasis activation markers [38].

Markers of haemostasis activation remain elevated several months after acute stroke [42] and are also demonstrable in patients with vascular dementia [43]. Elevated level of D-dimer after acute ischemic stroke identify patients with increased risk of mortality [44] and are a significant predic-

Pathophysiol Haemost Thromb 2003/2004;33:302-308 
tor of recurrent stroke in patients with previous stroke [45]. In the Edinburgh Artery Study a positive association of Ddimer level with the incidence of stroke among healthy men was also demonstrated [46]. Similarly, increased levels of F1+2 were found to be an independent predictor of subsequent cerebral and cardiac ischemic events in patients with previous transient ischemic attack [47].

\section{Activation Markers and Peripheral Arterial Disease}

Patients with peripheral arterial disease often have coexisting coronary and cerebrovascular disease and are at high risk of death not only from deterioration of limb ischemia but also from premature ischemic heart disease and stroke. Several studies have demonstrated elevated levels of haemostasis activation markers including F1+2, TAT and Ddimer in patients with peripheral arterial disease $[11,48]$. In these patients high level of D-dimer was associated with the extent of lower extremity arterial obstruction as measured by the ankle brachial index and were independently related to a higher degree of functional impairment [49]. Higher level of D-dimer has also been found in patients suffering thrombotic reocclusion following femoropopliteal artery angioplasty when compared with patients with maintained patency of the dilatated arterial segment [50]. Additionally, several studies have suggested a positive association between increased D-dimer concentration and future risk of major cardiovascular events, again suggesting the association between hypercoagulability and adverse outcomes in patients with arterial disease [12,51].

\section{Methodological Aspects}

With haemostasis activation markers one can follow the haemostatic process leading to thrombus formation from initiation, through amplification and simultaneous regulation, and ultimately do dissolution. Besides their potential prognostic and diagnostic value, they could also be useful in assessing the impact of various therapies. However, it should be kept in mind that measurement of haemostasis activation markers has several important biological and methodological disadvantages. Activation markers reflect the presence of thrombosis in any vascular bed, so they are not specific for e.g. thrombosis in the coronary circulation. Furthermore, elevations occur not only in the presence of overt thrombosis, but also during the hypercoagulable state. The cut-off level for each marker to be used for the definition of elevations is still largely unknown due to the use of different analytical methods, none of which have been standardized until now.

Many haemostasis activation markers are extremely sen- sitive to in vitro artefacts. Consequently, great care is necessary to attain valid samples. Phlebotomy must be preformed flawlessly, preferably from a fresh venepuncture, with the first $\mathrm{ml}$ of blood discarded. The samples must be drawn into appropriate anticoagulant or antiprotease cocktails and centrifugation must be performed at the appropriate temperature and g-force minutes, depending on the analyte of interest. Separation of plasma must be carefully performed, avoiding cellular contamination of the samples. Any incorrect procedure in blood collection and plasma separation may lead to falsely elevated levels. If assays of the specimens are not performed immediately, they should be aliquoted into vials with tight seals and stored frozen at $-20^{\circ}$ $\mathrm{C}$ or lower. Samples should subsequently be thawed in a 370 $\mathrm{C}$ water bath taking care not to let the sample temperature rise above $4^{\circ} \mathrm{C}$. When these assays are used in large clinical trials, care must be taken to train the personnel drawing and processing the samples and to record problems arising with phlebotomy or processing. These records should be periodically reviewed with the relevant personnel [52].

Since the initial development of a radioimmunoassay for determination of $\mathrm{F} 1+2$, later measurements of $\mathrm{F} 1+2$ have been performed by several enzyme-linked immunoassays. The F1+2 assay has been considered by many authors as an important test for the diagnosis of prethrombotic and thrombotic states, but interpretation of the results has been hampered by the dissimilarity of the assays with regard to the anticoagulants and antibodies used [53]. As for other haemostasis activation markers, appropriate blood sampling and processing is of utmost importance [54].

Measurement of TAT may be clinically useful as a marker for thrombin activity, because of its rapid turnover and because TAT is influenced to a smaller degree by preanalytical factors such as the technique of blood sampling than other haemostasis activation markers. Therefore, TAT has been adopted in a number of clinical investigations and represents a diagnostic tool for the detection of hypercoagulability [55].

Since the normal FPA concentration is around or below $2 \mathrm{ng} / \mathrm{mL}$ the only sufficiently sensitive methods are radioimmunoassay or enzyme-linked immunoassay, neither of them being suitable for bedside clinical application because of the very long turn-around times. Furthermore, measurement of FPA requires meticulous acquisition, collection, and processing of blood samples to avoid thrombin elaboration and ex vivo increases in FPA concentration [5]. The very short plasma half-life of FPA additionally limits its clinical utility. However it can also be measured in urine collected over 24 hours. Urinary excretion is rapid, therefore measurement of FPA in the urine reflects the mean of activity over the collection period [23]. 
To overcome limitations in measuring FPA, measurement of soluble fibrin has been proposed. The advantage of measuring soluble fibrin over FPA to detect the action of thrombin on fibrinogen is the considerably longer half-life of soluble fibrin (several hours) in the circulation. However, the molecular structure of soluble fibrin species in plasma has not been well characterized, and the available assays differ considerably in the methodology used for detecting soluble fibrin. The most widely used assays for soluble fibrin are enzyme-linked immunoassays. These tests typically incorporate capture monoclonal antibodies that are specific for neoepitopes of soluble fibrin exposed on the fibrinogen molecule after cleavage of fibrinopeptides [30]. Only a small number of soluble fibrin assays have entered clinical evaluation or are available commercially. Further clinical studies are needed to establish the clinical utility of specific soluble fibrin assays. A clear-cut clinical advantage of these assays over D-dimer or similar assays remains to be established [32].

Development of monoclonal antibodies specific for Ddimer has permitted accurate quantification of cross linked fibrin degradation products in a variety of assay formats [56]. D-dimer is relatively stable in plasma samples, which facilitates their use in clinical and epidemiological studies. D-dimer is also widely available in many hospitals for the diagnosis of disseminated intravascular coagulation and venous thromboembolism. Therefore, D-dimer is the haemostasis activation marker, whose diagnostic and prognostic value in cardiovascular disease has been studied most intensively.

\section{Conclusions}

The haemostatic system plays a critical role in the genesis of atherosclerosis and in the generation of the thrombus that causes the most characteristic end point of cardiovascular disease. Today a large number of markers are available to determine the pathophysiological alterations occurring in patients with cardiovascular disease. These markers are used to characterize different pathways and to develop a better understanding of the ongoing processes in patients with cardiovascular disease. Among activation markers of coagulation and fibrinolysis the prognostic value of D-dimer has been best characterized. There is substantial evidence that D-dimer is a strong, consistent predictor of cardiovascular events in subjects with or without clinically detectable arterial disease. The prognostic value of other markers of activation is rather controversial and remains to be fully defined in future studies.

\section{Acknowledgement}

Financial support of the Slovene Ministry of Education, Science and Sport is gratefully acknowledged (grant no. J33412).

\section{References}

1 Fuster V, Badimon L, Badimon JJ, Chesebro JH. The pathogenesis of coronary artery disease and the acute coronary syndromes $\mathrm{N}$ Engl $\mathrm{J}$ Med 1992;326:310-318.

- 2 Fareed J, Hoppensteadt DA, Leya F, Iqbal O, Wolf H, Bick R. Useful laboratory tests for studying thrombogenesis in acute cardiac syndromes. Clin Chem 1998;44:1845-1853.

3 Haeberli A. Prothrombin fragment F1+2. In: Jespersen J, Bertina RM, Haverkater F (eds): Laboratory techniques in thrombosis - A manual. Dordrecht-Boston-London, Kluwer, 1999, pp 217222.

4 Lau HK, Rosenberg RD. The isolation and characterization of a specific antibody population directed against the thrombin antithrombin complex. J Biol Chem 1980;255:5885-5893.

5 Haeberli A. Fibrinopeptide A (FPA). In: Jespersen J, Bertina RM, Haverkater F (eds): Laboratory techniques in thrombosis - A manual. DordrechtBoston-London, Kluwer, 1999, pp 199-207.

6 Wilf J, Minton AP. Soluble fibrin-fibrinogen complexes as intermediates in fibrin gel formation. Biochemistry 1986;25:3124-3133.
Gaffney PJ, Edgell T, Creighton-Kempsford LJ, Wheeler S, Tarelli E. Fibrin degradation product (FnDP) assays: analysis of standardization issues and target antigens in plasma. $\mathrm{Br} \mathrm{J}$ Haematol 1995;90:187-194.

Ridker PM, Hennekens CH, Cerskus A, Stampfer MJ. Plasma concentration of cross-linked fibrin degradation product (D-dimer) and the risk of future myocardial infarction among apparently healthy men. Circulation 1994;90:2236-2240. Levi M, de Boer JP, Roem D, ten Cate JW, Hack CE. Plasminogen activation in vivo upon intravenous infusion of DDAVP. Quantitative assessment of plasmin-alpha 2-antiplasmin complex with a novel monoclonal antibody based radioimmunoassay. Thromb Haemost 1992;67:111-116.

10 Astrup T, Permin TM. Fibrinolysis in the animal organism. Nature 1947; 159: 681-682.

11 van der Bom JG, Bots ML, Haverkate F, Meijer P, Hofman PA, Kluft C , Grobbee DE. Activation products of the haemostatic system in coronary, cerebrovascular and peripheral arterial disease. Thromb Haemost 2001;85:234-239.
12 Fowkes FG, Lowe GD, Housley E, Rattray A, Rumley A, Elton RA, MacGregor IR, Dawes J. Cross-linked fibrin degradation products, progression of peripheral arterial disease, and risk of coronary heart disease. Lancet 1993;342:84-86.

3 Koenig W, Rothenbacher D, Hoffmeister A, Griesshammer M, Brenner H. Plasma fibrin Ddimer levels and risk of stable coronary artery disease: results of a large case-control study. Arterioscler Thromb Vasc Biol 2001;21:17011705.

14 Heinrich J, Schulte H, Schonfeld R, Kohler E, Assmann G. Association of variables of coagulation, fibrinolysis and acute-phase with atherosclerosis in coronary and peripheral arteries and those arteries supplying the brain. Thromb Haemost 1995;73:374-379.

15 Danesh J, Whincup P, Walker M, Lennon L, Thomson A, Appleby P, Rumley A, Lowe GD. Fibrin D-dimer and coronary heart disease: prospective study and meta-analysis. Circulation 2001;103:2323-2327. 
16 Lowe GD, Rumley A, Sweetnam PM, Yarnell JW, Rumley J. Fibrin D-dimer, markers of coagulation activation and the risk of major ischaemic heart disease in the Caerphilly study. Thromb Haemost 2001;86:822-827.

17 Folsom AR, Aleksic N, Park E, Salomaa V, Juneja $\mathrm{H}$, Wu KK. Prospective study of fibrinolytic factors and incident coronary heart disease: the Atherosclerosis Risk in Communities (ARIC) Study. Arterioscler Thromb Vasc Biol 2001;21:611-617.

18 Cushman M, Lemaitre RN, Kuller LH, Psaty BM, Macy EM, Sharrett AR, Tracy RP. Fibrinolytic activation markers predict myocardial infarction in the elderly. The Cardiovascular Health Study. Arterioscler Thromb Vasc Biol 1999;19:493-498.

19 Hoffmeister HM, Jur M, Wendel HP, Heller W, Seipel L. Alterations of coagulation and fibrinolytic and kallikrein-kinin systems in the acute and postacute phases in patients with unstable angina pectoris. Circulation 1995; 91:2520-2527.

20 Biasucci LM, Liuzzo G, Caligiuri G, Quaranta G, Andreotti F, Sperti G, et al. Temporal relation between ischemic episodes and activation of the coagulation system in unstable angina. Circulation 1996; 93: 2121-2127.

-21 Merlini PA, Bauer KA, Oltrona L, Ardissino D, Cattaneo M, Belli C, Mannucci PM, Rosenberg RD. Persistent activation of coagulation mechanism in unstable angina and myocardial infarction. Circulation 1994;90:61-68.

22 Granger CB, Becker R, Tracy RP, Califf RM, Topol EJ, Pieper KS, Ross AM, Roth S, Lambrew C, Bovill EG. Thrombin generation, inhibition and clinical outcomes in patients with acute myocardial infarction treated with thrombolytic therapy and heparin: results from the GUSTO-I Trial. GUSTO-I Hemostasis Substudy Group. Global Utilization of Streptokinase and TPA for Occluded Coronary Arteries. J Am Coll Cardiol 1998;31:497-505.

23 Ardissino D, Gamba MG, Merlini PA, Rolla A Barberis P, Demicheli G, Testa S, Bruno N, Specchia G. Fibrinopeptide A excretion in urine: a marker of the cumulative thrombin activity in stable versus unstable angina patients. Am J Cardiol 1991;68:58B-63B.

24 Eisenberg PR, Kenzora JL, Sobel BE, Ludbrook PA, Jaffe AS. Relation between ST segment shifts during ischemia and thrombin activity in patients with unstable angina. J Am Coll Cardiol 1991;18:898-903.

25 Wilensky RL, Bourdillon PD, Vix VA, Zeller JA Intracoronary artery thrombus formation in unstable angina: a clinical, biochemical and angiographic correlation. J Am Coll Cardiol 993;21:692-699.

-26 Ardissino D, Merlini PA, Gamba G, Barberis P, Demicheli G, Testa S, Colombi E, Poli A, Fetiveau R, Montemartini C. Thrombin activity and early outcome in unstable angina pectoris. Circulation 1996;93:1634-1639.

27 Li YH, Teng JK, Tsai WC, Tsai LM, Lin LJ, Guo HR, Chen JH. Prognostic significance of elevated hemostatic markers in patients with acute myocardial infarction. J Am Coll Cardiol 1999;33:15431548.

28 Bayes-Genis A, Mateo J, Santalo M, Oliver A, Guindo J, Badimon L, Martinez-Rubio A, Fontcuberta J, Schwartz RS, De Luna AB. DDimer is an early diagnostic marker of coronary ischemia in patients with chest pain. Am Heart $\mathrm{J}$ 2000;140:379-384.
29 Figueras J, Monasterio Y, Lidon RM, Nieto E, Soler-Soler J. Thrombin formation and fibrinolytic activity in patients with acute myocardial infarction or unstable angina: in-hospital course and relationship with recurrent angina at rest. J Am Coll Cardiol 2000;36:2036-2043.

30 Lee LV, Ewald GA, McKenzie CR, Eisenberg PR. The relationship of soluble fibrin and cross-linked fibrin degradation products to the clinical course of myocardial infarction. Arterioscler Thromb Vasc Biol 1997;17:628-633.

31 Marder VJ, Zareba W, Horan JT, Moss AJ, Kanouse JJ. Automated latex agglutination and ELISA testing yield equivalent D-dimer results in patients with recent myocardial infarction. THROMBO Research Investigators. Thromb Haemost 1999;82:1412-1416.

32 Dempfle CE, Kontny F, Abildgaard U. Predictive value of coagulation markers concerning clinical outcome 90 days after anterior myocardial infarction. Thromb Haemost 1999;81:701-704.

33 Galvani M, Abendschein DR, Ferrini D, Ottani F, Rusticali F, Eisenberg PR. Conjunctive administration of intravenous heparin attenuates crosslinked fibrin degradation in patients treated with streptokinase. Thromb Haemost 1996;76:339-343. 34 Hetland O, Knudsen A, Dickstein K, Nilsen DW. Characteristics and prognostic impact of plasma fibrin monomer (soluble fibrin) in patients with coronary artery disease. Blood Coagul Fibrinolysis 2002;13:301-308.

35 Kumagai K, Fukunami M, Ohmori M, Kitabatake A, Kamada T, Hoki N. Increased intracardiovascular clotting in patients with chronic atrial fibrillation. J Am Coll Cardiol 1990;16:377-380.

36 Lip GYH, Lowe GDO, Rumley A, Dunn FG. Increased markers of thrombogenesis in chronic atrial fibrillation: effects of warfarin treatment. $\mathrm{Br}$ Heart J 1995;73:527-533.

37 Vene N, Mavri A, Kosmelj K, Stegnar M. High Ddimer levels predict cardiovascular events in patients with chronic atrial fibrillation during oral anticoagulant therapy. Thromb Haemost 2003;90:1163-1172.

38 Ageno W, Finazzi S, Steidl L, Biotti MG, Mera V, Melzi D'Eril G, Venco A. Plasma measurement of D-dimer levels for the early diagnosis of ischemic stroke subtypes. Arch Intern Med 2002;162:25892593.

39 Barber M, Langhorne P, Rumley A, Lowe GD, Stott DJ. Hemostatic function and progressing ischemic stroke: D-dimer predicts early clinical progression. Stroke 2004;35:1421-1425.

40 Yamazaki M, Uchiyama S, Maruyama S. Alterations of haemostatic markers in various subtypes and phases of stroke. Blood Coagul Fibrinolysis 1993;4:707-712.

41 Broderick JP, Phillips SJ, O'Fallon WM, Frye RL, Whisnant JP. Relationship of cardiac disease to stroke occurrence, recurrence, and mortality. Stroke 1992;23:1250-1256.

42 Reganon E, Vila V, Martinez-Sales V, Vaya A, Lago A, Alonso P, Aznar J. Association between inflammation and hemostatic markers in atherothrombotic stroke. Thromb Res 2003;112:217-221.

-43 Stott DJ, Spilg E, Campbell AM, Rumley A, Mansoor MA, Lowe GD. Haemostasis in ischaemic stroke and vascular dementia. Blood Coagul Fibrinolysis 2001;12:651-657.

44 Feinberg WM, Erickson LP, Bruck D, Kittelson J. Hemostatic markers in acute ischemic stroke. Association with stroke type, severity, and outcome. Stroke 1996;27:1296-1300.
45 Di Napoli M, Papa F; Villa Pini Stroke Data Bank Investigators. Inflammation, hemostatic markers, and antithrombotic agents in relation to long-term risk of new cardiovascular events in first-ever ischemic stroke patients. Stroke 2002;33:17631771.

46 Smith FB, Lee AJ, Fowkes FG, Price JF, Rumley A, Lowe GD. Hemostatic factors as predictors of ischemic heart disease and stroke in the Edinburgh Artery Study. Arterioscler Thromb Vasc Biol 1997; 17:3321-335.

47 Côté R, Wolfson C, Solymoss S, Mackey A, Leclerc JR, Simard D, et al. Hemostatic markers in patients at risk of cerebral ischemia. Stroke 2000;31:1856-1862.

48 Herren T, Stricker H, Haeberli A, Do DD, Straub PW. Fibrin formation and degradation in patients with arteriosclerotic disease. Circulation 1994;90:2679-2686.

49 McDermott MM, Greenland P, Green D, Guralnik JM, Criqui MH, Liu K, Chan C, et al. D-dimer, inflammatory markers, and lower extremity functioning in patients with and without peripheral arterial disease. Circulation 2003;107:3191-3198.

50 Jorgensen B, Nielsen JD, Norgard J, Helligso P, Baekgaard N, Egeblad M. Cross-linked fibrin degradation products (XL-FDP) as marker of early rethrombosis in percutaneous transluminal angioplasty. Eur J Vasc Surg 1993;7:720-724.

51 Komarov A, Panchenko E, Dobrovolsky A, Karpov Y, Deev A, Titaeva E, et al. D-dimer and platelet aggregability are related to thrombotic events in patients with peripheral arterial occlusive disease. Eur Heart J 2002;16:1309-1316.

52 Becker RC, Bovill EG, Seghatchian MJ, Samama MM. Pathobiology of thrombin in acute coronary syndromes. Am Heart J 1998;136:S19-31.

53 Tripodi A, Chantarangkul V, Bottasso B, Mannucci PM. Poor comparability of prothrombin fragment $1+2$ values measured by two commercial ELISA methods: influence of different anticoagulants and standards. Thromb Haemost 1994;71:605-608.

54 Greenberg CS, Hursting MJ, Macik BG, Ortel TL, Kane WH, Moore BM. Evaluation of preanalytical variables associated with measurement of prothrombin fragment 1.2. Clin Chem 1994;40:19621969.

55 Seitz R, Blanke H, Pratorius G, Strauer BE, Egbring R. Increased thrombin activity during thrombolysis. Thromb Haemost 1988;59:541-542. 56 Rylatt DB, Blake AS, Cottis LE, Massingham DA, Fletcher WA, Masci PP, Whitaker AN, Elms M, Bunce I, Webber AJ, et al. An immunoassay for human D dimer using monoclonal antibodies. Thromb Res 1983;31:767-778. 